\title{
Can clinical response to cyclosporin in chronic severe asthma be predicted by an in vitro T-lymphocyte proliferation assay?
}

\author{
A.G. Alexander*, N.C. Barnes**, A.B. Kay*, C.J. Corrigan*
}

Can clinical response to cyclosporin in chronic severe asthma be predicted by an in vitro T-lymphocyte proliferation assay? A.G. Alexander, N.C. Barnes, A.B. Kay, C.J. Corrigan. (CERS Journals Ltd 1996.

ABSTRACT: This study tests the hypothesis that the clinical response to cyclosporin therapy of patients with chronic severe asthma is related to the sensitivity of their T-lymphocytes to the antiproliferative effects of cyclosporin in vitro. In a previous study, we observed such a relationship with glucocorticoids and the same lectindriven proliferation assay was used in the present study.

Peripheral blood mononuclear cells were obtained from 33 patients participating in a cross-over trial of oral cyclosporin therapy during both cyclosporin and placebo treatment periods, and cultured in the presence of phytohaemagglutinin and serial dilutions of cyclosporin and dexamethasone. Proliferation was measured by tritiated thymidine uptake.

Both cyclosporin and dexamethasone inhibited T-lymphocyte proliferation in a concentration-dependent manner in vitro at concentrations encompassing those achieved in peripheral blood during therapy in vivo. T-lymphocytes from the asthmatic patients showed a range of sensitivity to the antiproliferative effects of cyclosporin, but this could not be correlated with improvements in peak expiratory flow rate (PEFR) or forced expiratory volume in one second (FEV1) during cyclosporin therapy as compared with placebo.

In contrast to previous observations with glucocorticoids, this in vitro T-lymphocyte proliferation assay is not predictive of clinical response to cyclosporin therapy in chronic severe asthmatics.

Eur Respir J., 1996, 9, 1421-1426.
*Dept of Allergy and Clinical Immunology, National Heart \& Lung Institute, London, UK. **Dept of Thoracic Medicine, London Chest Hospital, London, UK.

Correspondence: C.J. Corrigan Dept of Allergy and Clinical Immunology National Heart \& Lung Institute

London SW3 6LY

UK

Keywords: Asthma

cyclosporin

glucocorticoid

proliferation assay

T-lymphocyte

Received: October 301995

Accepted after revision March 251996
Glucocorticoids, by inhaler and when necessary systemically, are the mainstay of treatment for asthma [1], but a subset of patients respond poorly or not at all to glucocorticoid therapy $[2,3]$. In a previous study, we classified a group of patients with chronic severe asthma as clinically glucocorticoid sensitive or resistant on the basis of changes in lung function after oral prednisolone therapy [3]. In a lectin-driven assay, dexamethasone at a therapeutic concentration $\left(10^{-7} \mathrm{~mol} \cdot \mathrm{L}^{-1}\right)$ inhibited the proliferation [3] of, and cytokine elaboration [4] by, peripheral blood T-lymphocytes from the sensitive, but not the resistant patients. In contrast, cyclosporin at a therapeutic concentration $\left(500 \mathrm{ng} \cdot \mathrm{mL}^{-1}\right)$ significantly inhibited both proliferation of and cytokine production by T-lymphocytes from the resistant as well as sensitive patients [4]. Taking the sensitive and resistant patients together, the clinical response of the patients to glucocorticoid therapy correlated with the degree of inhibition of their peripheral blood T-lymphocytes by glucocorticoids in vitro [3].

In a recent double-blind, cross-over trial, we showed that cyclosporin therapy for 12 weeks resulted in a mean increase in peak expiratory flow rate (PEFR) of $12 \%$ as compared with placebo therapy $(\mathrm{p}<0.004)$ in patients with oral glucocorticoid-dependent chronic severe asthma [5]. A spectrum of clinical response was observed, with some patients showing an increase in PEFR of greater than $50 \%$ as compared with placebo, whilst others did not improve at all.

By analogy with glucocorticoids, we sought to obtain evidence, prospectively, that any clinical improvement of the asthmatic patients with cyclosporin therapy might be related to T-lymphocyte inhibition. We hypothesized that peripheral blood T-lymphocytes from these patients show a range of sensitivity to the antiproliferative effects of cyclosporin in vitro, and that the degree of inhibition of T-lymphocyte proliferation can be correlated with the extent of the clinical response to oral cyclosporin therapy. It might then be possible to use the T-lymphocyte proliferation assay to identify, prospectively, patients likely to respond clinically to cyclosporin therapy. We also wished to investigate our previous observation that the sensitivity of T-lymphocytes to inhibition by cyclosporin and dexamethasone are not linearly related, and whether the antiproliferative effects of the two drugs in vitro were influenced by oral administration of cyclosporin in vivo.

\section{Methods}

\section{Patients}

The clinical trial investigating the efficacy of cyclosporin therapy in asthma has been described in detail 
Table 1. - Baseline (pretrial) patient characteristics, treatment and respiratory function data

\begin{tabular}{|c|c|}
\hline \multirow{2}{*}{$\begin{array}{l}\text { Sex M/F } \\
\text { Age yrs* }\end{array}$} & $13 / 20$ \\
\hline & $(21-64)$ \\
\hline Duration of asthma yrs* & $(5-54)$ \\
\hline Skin prick test positive $\mathrm{e}^{\#} \mathrm{n}$ & 20 \\
\hline $\operatorname{IgE~IU} \cdot \mathrm{mL}^{-1 *}$ & $146(6-1250)$ \\
\hline Continuous oral prednisolone yrs* & $9.3 \quad(0.3-25)$ \\
\hline Prednisolone dosage $\mathrm{mg} \cdot \mathrm{day}^{-1 *}$ & $8.5 \quad(5-20)$ \\
\hline Inhaled glucocorticoid usage $\mu \mathrm{g} \cdot$ day $^{-1+}$ & $1665(90)$ \\
\hline Oral theophylline usage $n$ & 23 \\
\hline $\begin{array}{ll}\text { PEFR } & \mathrm{L} \cdot \mathrm{min}^{-1+} \\
\% \text { pred }\end{array}$ & $\begin{array}{ll}239 & (19.0) \\
54 & \end{array}$ \\
\hline $\begin{array}{ll}\mathrm{FEV}_{1} & \mathrm{~L}^{+} \\
& \% \text { pred }\end{array}$ & $\begin{array}{l}1.73(0.14) \\
60\end{array}$ \\
\hline $\begin{array}{ll}\mathrm{VC} & \mathrm{L}^{+} \\
& \% \text { pred }\end{array}$ & $\begin{array}{ll}3.09 & (0.21) \\
83 & \end{array}$ \\
\hline
\end{tabular}

*: data presented as mean and range in parenthesis; ${ }^{+}$: data presented as mean and SEM in parenthesis; \#: defined as one or more positive skin-prick tests to a range of 12 common aeroallergens in the presence of a positive histamine control and a negative vehicle control. M: male; F: female; IgE: immunoglobulin E; PEFR: peak expiratory flow rate; FEV1: forced expiratory volume in one second; VC: vital capacity; \% pred: percentage of predicted value.

previously [5]. Briefly, documented chronic asthmatics aged 18-65 yrs who had a forced expiratory volume in one second (FEV1) and/or PEFR below $75 \%$ of the predicted value and $>20 \%$ reversibility to $\beta_{2}$-agonist were considered for inclusion in the trial if they required longterm maintenance treatment with 5-20 mg oral prednisolone daily in addition to maximal, tolerated and effective other therapy, including high-dose inhaled glucocorticoids (table 1).

Written informed consent was obtained from each patient and the study was approved by the Ethics Committee of the Royal Brompton National Heart and Lung Hospitals.

\section{Study design}

After a 4 week run-in period, 33 subjects were randomized to receive cyclosporin or matching placebo for 12 weeks in addition to their usual treatment. Following a 2 week wash-out period, subjects crossed over to placebo or cyclosporin medication for a further 12 weeks. The cross-over design allows direct intrasubject comparisons, with each patient acting as his/her own control. For this reason, all treatment except inhaled bronchodilator usage was kept constant during the trial, although during exacerbations oral prednisolone dosage was increased if required. Exacerbations were defined as worsening of asthma symptoms in association with decreased PEFR, and were treated by increasing the prednisolone dosage. In view of the previously described effects both of disease exacerbation and oral prednisolone therapy on T-lymphocyte activation in vivo [6, 7], patients whose prednisolone dosage had been increased during a disease exacerbation and at the time of blood sampling were receiving prednisolone at a greater dosage than maintenance were excluded from the study.

Patients attended the clinic weekly for the first 4 weeks of each treatment period and, thereafter, every 2 weeks. Cyclosporin was started at $5 \mathrm{mg} \cdot \mathrm{kg}^{-1}$ daily and, thereafter, dosage was adjusted to achieve whole blood concentrations of 100-200 $\mathrm{ng} \cdot \mathrm{mL}^{-1}$, measured using a specific monoclonal radioimmunoassay (Cyclo-Trac SP; Incstar Corp., Stillwater MN, USA). The mean concentrations of all patients during weeks 9-12 of cyclosporin therapy was 151.7 (SEM 7.2) $\mathrm{ng} \cdot \mathrm{mL}^{-1}$ [5].

\section{Materials}

Stock solutions were prepared as follows and the same batches used for all experiments. Appropriate dilutions were made up in RPMI-1640 (Gibco, Paisley, Scotland) containing $2 \mathrm{mmol} \cdot \mathrm{L}^{-1} \mathrm{~L}$-glutamine for individual experiments.

Phytohaemagglutinin (PHA) (Sigma, Poole, UK) was prepared at a concentration of $100 \mu \mathrm{g} \cdot \mathrm{mL}^{-1}$ in RPMI1640 and filtered sterile. This was stored in aliquots at $-20^{\circ} \mathrm{C}$ and was added to cultured cells at a final concentration of $5 \mu \mathrm{g} \cdot \mathrm{mL}^{-1}$.

Dexamethasone (Sigma) was dissolved in ethanol to a concentration of $2 \times 10^{-4} \mathrm{~mol} \cdot \mathrm{L}^{-1}$ and stored at $-20^{\circ} \mathrm{C}$. In use, RPMI-1640 was added to produce a final stock concentration of $2 \times 10^{-6} \mathrm{~mol} \cdot \mathrm{L}^{-1}$.

Cyclosporin oily suspension $\left(100 \mathrm{mg} \cdot \mathrm{mL}^{-1}\right)$ (Sandoz Pharmaceuticals) was dispersed with thorough mixing in RPMI-1640 to a concentration of $10 \mu \mathrm{g} \cdot \mathrm{mL}^{-1}$ and stored in aliquots at $-20^{\circ} \mathrm{C}$. Cyclosporin oily suspension vehicle (Sandoz Pharmaceuticals) was diluted in the same way.

Serial dilutions of drugs were made at the time of each experiment by dissolving the stock solutions in RPMI1640 supplemented with $2 \mathrm{mmol} \cdot \mathrm{L}^{-1} \mathrm{~L}$-glutamine. Solutions were filtered sterile through a $0.22 \mu \mathrm{m}$ pore size filter as appropriate.

\section{Peripheral blood mononuclear cell isolation and culture}

Fifty millilitres of peripheral venous blood were obtained from each patient at the same time of day at a clinic visit during the last 4 weeks both of the cyclosporin and placebo treatment periods, a mean of 17.0 (SEM 0.44) $\mathrm{h}$ after the previous cyclosporin (or placebo) dose. The blood was added to preservative-free heparin in a sterile fashion and then mixed with an equal volume of RPMI1640 medium. Thirty millilitre aliquots were layered onto $20 \mathrm{~mL}$ aliquots of Ficoll-Paque (Pharmacia, Uppsala, Sweden) in $50 \mathrm{~mL}$ sterile conical tubes (Falcon, Becton Dickinson, Cowley, UK). After centrifugation at $400 \times \mathrm{g}$ for $20 \mathrm{~min}$ at $20^{\circ} \mathrm{C}$, peripheral blood mononuclear cells (PBMCs) were removed from the plasma/Ficoll interface with gentle suction, transferred to sterile polystyrene universal containers, and washed three times with RPMI1640. Cell manipulations were performed at $4^{\circ} \mathrm{C}$.

PBMCs were resuspended in RPMI-1640 supplemented with $2 \mathrm{mmol} \cdot \mathrm{L}^{-1} \mathrm{~L}$-glutamine at $4 \times 10^{6}$ cells $\cdot \mathrm{mL}^{-1}$. Seventy five microlitre aliquots of this cell suspension, with or without added PHA, were added to $75 \mu \mathrm{L}$ aliquots of serially diluted cyclosporin and dexamethasone solutions or medium control in triplicate in sterile 96-well round-bottomed culture plates (Cel-cult; Sterilin, Hounslow, UK). The total volume in each well was $150 \mu \mathrm{L}$, with final concentrations of the cell suspension of $2 \times 10^{6}$ cells $\cdot \mathrm{mL}^{-1}$, PHA of $5 \mu \mathrm{g} \cdot \mathrm{mL}^{-1}$, and drug concentrations in the range $25-5,000 \mathrm{ng} \cdot \mathrm{mL}^{-1}$ for cyclosporin and $10^{-9}$ $10^{-6} \mathrm{~mol} \cdot \mathrm{L}^{-1}$ for dexamethasone. 
Culture plates were incubated for $72 \mathrm{~h}$ in a humidified atmosphere with 5\% carbon dioxide. T-lymphocyte proliferation was measured by uptake of tritiated methylthymidine. Sterile tritiated thymidine solution (Amersham, Amersham, UK) $\left(24.6 \mathrm{kBq} \cdot\right.$ well $^{-1}$ in a volume of $10 \mu \mathrm{L})$ was added to cell culture wells for the last $6-8$ $\mathrm{h}$ of the $72 \mathrm{~h}$ incubation period. After incubation, cells were harvested onto glass-fibre filter paper with a cell harvester apparatus (Ilacon, Tonbridge, UK), and the incorporated radiolabel was counted with a beta-spectrometer. After the geometric mean of the triplicate counts was calculated, results were expressed as percentages of incorporated counts in the presence of drugs as compared with the medium only (positive) control. Doseresponse curves were constructed for suppression of T-lymphocyte proliferation by cyclosporin and by dexamethasone.

\section{Statistical analysis}

Analysis of the clinical trial has been reported previously [5], but briefly, daily morning prebronchodilator PEFR data for each patient were summarized as a mean for the last 4 weeks of each treatment period. Clinical improvement in mean PEFR was determined as percentage difference between cyclosporin therapy and placebo, i.e. $(($ cyclosporin/placebo $) \times 100)-100$.

Dose-response data for the antiproliferative effects of cyclosporin and dexamethasone on T-lymphocytes from the asthmatic patients are expressed as the mean (SEM) percentages of the proliferation observed in the presence of the medium control for each treatment period (fig. 1). These data conformed to a Gaussian distribution at each drug concentration.

In vitro concentrations of $250 \mathrm{ng} \cdot \mathrm{mL}^{-1}$ cyclosporin and $10^{-7} \mathrm{~mol} \cdot \mathrm{L}^{-1}$ dexamethasone were used in analysis of the antiproliferative effects of these drugs on PBMC

a)

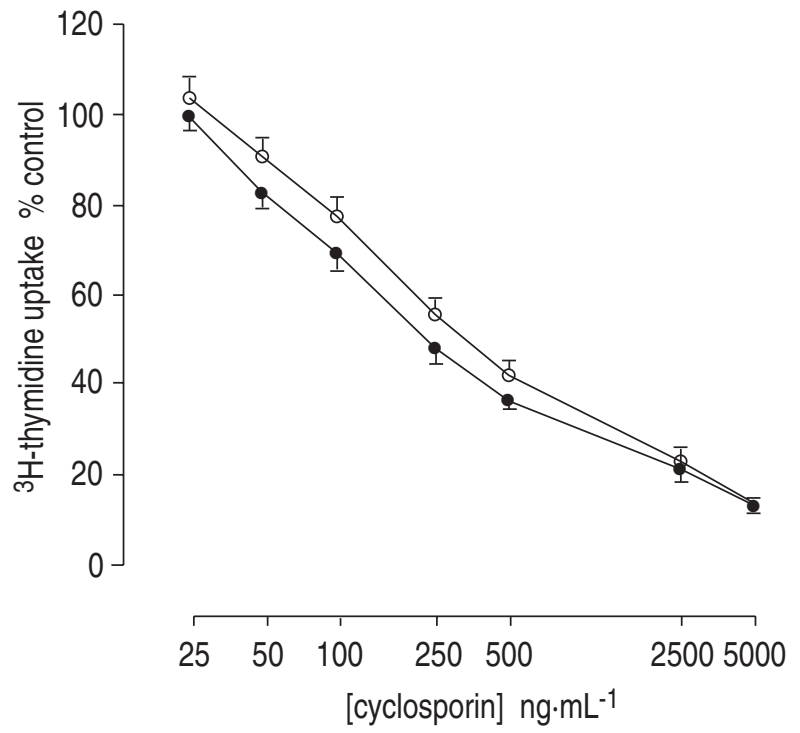

in vitro as they most closely represent the therapeutic concentrations achieved in these patients in vivo during cyclosporin [5] and glucocorticoid [3] therapy, respectively. Between-drug and between-treatment comparisons were made using the paired t-test. Subgroup comparisons of atopic status and of theophylline usage were made using the two sample t-test. Probability values for correlation coefficients were calculated using Pearson's test. A two-tailed probability of $5 \%$ or less was considered statistically significant. Analysis was performed using the Minitab statistical software package (State College, Pennsylvania, USA).

\section{Results}

Thirty patients completed the clinical trial [5], one failing to cross over to cyclosporin therapy and two to placebo. Four patients were excluded during placebo and three during cyclosporin therapy since at the proposed time of blood sampling they were receiving prednisolone at increased dosage during a disease exacerbation (see Methods). Cell culture was unsuccessful for one patient during placebo and three during cyclosporin therapy, and for one patient during placebo therapy no data for dexamethasone inhibition were available. Data are, therefore, available for analysis for 26 patients during placebo and 26 patients during cyclosporin therapy. For between-treatment comparisons, paired data from both treatment periods were available for cyclosporin for 19 patients and for both drugs for 18 patients.

Addition of cyclosporin and dexamethasone to PHAstimulated peripheral blood T-lymphocytes from the 26 asthmatic patients described above in the concentration ranges $25-5,000 \mathrm{ng} \cdot \mathrm{mL}^{-1}\left(2.1 \times 10^{-8}-4.2 \times 10^{-6} \mathrm{~mol} \cdot \mathrm{L}^{-1}\right)$ and $10^{-9}-10^{-6} \mathrm{~mol} \cdot \mathrm{L}^{-1}$, respectively, resulted in dosedependent suppression of proliferation (fig. 1). No proliferation was observed in the absence of PHA. Viability

b)

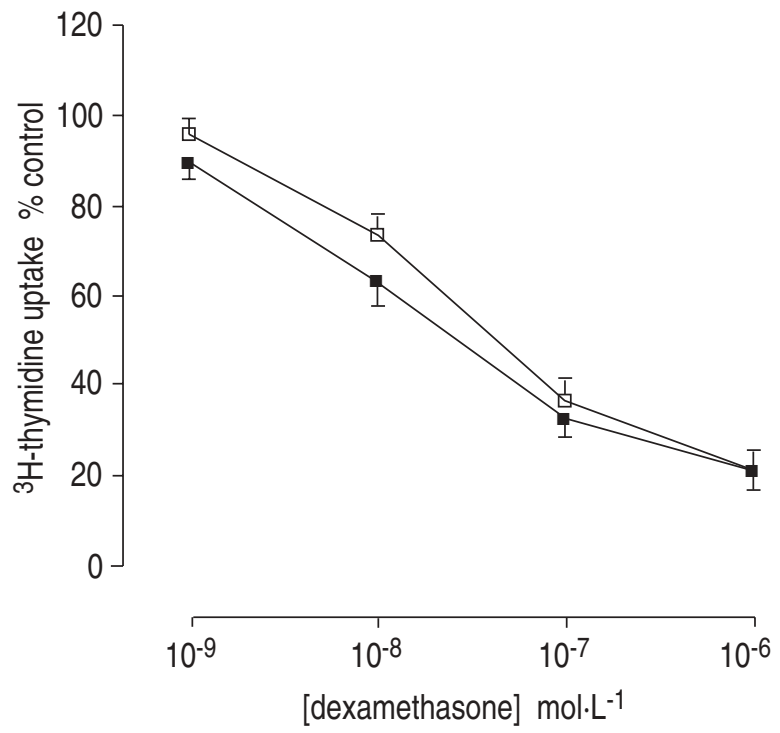

Fig. 1. - Dose-response curves for mean inhibition by: a) cyclosporin; and b) dexamethasone of in vitro PHA-induced proliferation of peripheral blood T-lymphocytes from 26 patients (see text) with chronic severe asthma during therapy with placebo (open symbols) and cyclosporin (closed symbols). Uptake of ${ }^{3} \mathrm{H}$-thymidine (counts per minute) is expressed as a percentage of that measured in the presence of the medium (no drug) control. Vertical bars represent the SEM. PHA: phytohaemagglutinin. 
studies with trypan blue exclusion showed no toxic effect of either drug at any concentration employed. The ethanol and cyclosporin vehicles showed no significant effect on lymphocyte proliferation at concentrations used in the lowest drug dilutions.

T-lymphocytes from the asthmatic patients showed a wide range of sensitivity to the antiproliferative effects of both cyclosporin at a concentration of $250 \mathrm{ng} \cdot \mathrm{mL}^{-1}$ (fig. 2a) and dexamethasone at $10^{-7} \mathrm{~mol} \cdot \mathrm{L}^{-1}$ (fig. 2b) in vitro. Although PBMCs from some of the asthmatic patients showed considerable variability in their susceptibility to the antiproliferative effects of cyclosporin in vitro when receiving cyclosporin as compared with placebo therapy (fig. 2a), there was no significant difference between the mean percentage inhibition of proliferation in each treatment period when the patients were considered as a group $(p=0.73)$. Similarly, no difference was observed with dexamethasone (fig. $2 b)(p=0.71)$, suggesting that oral cyclosporin therapy did not influence, in a consistent manner, the sensitivity of PBMCs to the antiproliferative effect of either cyclosporin or dexamethasone in vitro. There were no significant differences between the antiproliferative effects of either cyclosporin or dexamethasone when atopic patients were compared with nonatopic patients, or according to oral theophylline usage (data not shown).

The sensitivity of PHA-activated peripheral blood Tlymphocytes from the asthmatic patients during placebo therapy to the antiproliferative effects of cyclosporin at an in vitro concentration of $250 \mathrm{ng} \cdot \mathrm{mL}^{-1}$, expressed as a percentage of the proliferation observed in the presence of medium control, did not correlate with the clinical response of the patients to cyclosporin therapy, as measured [5] by percentage increases in either morning prebronchodilator PEFR $(p=0.47)$ or FEV1 $(p=0.14)$ during cyclosporin therapy as compared with placebo.

No correlation was observed between the antiproliferative effects of cyclosporin at $250 \mathrm{ng} \cdot \mathrm{mL}^{-1}$ and dexamethasone at $10^{-7} \mathrm{~mol} \cdot \mathrm{L}^{-1}$ on $\mathrm{T}$-lymphocytes from the asthmatic patients obtained during the placebo treatment period.

a)

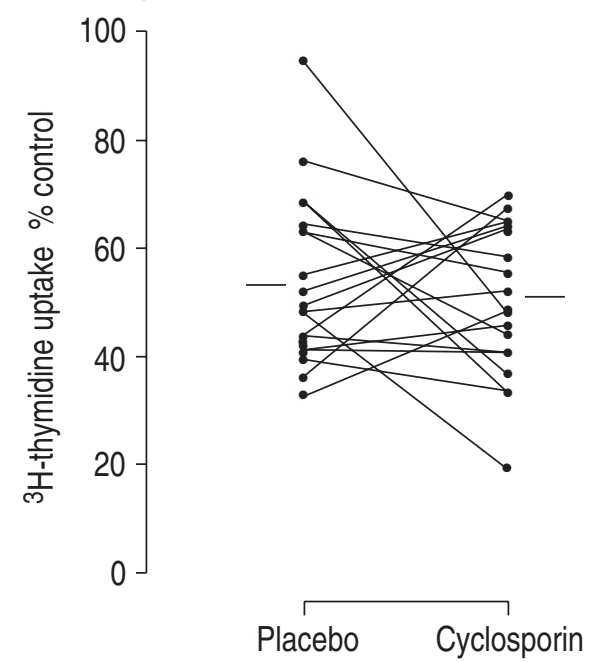

\section{Discussion}

In this study, we have demonstrated a range of sensitivity of PHA-activated peripheral blood T-lymphocytes from patients with glucocorticoid-dependent chronic severe asthma to the antiproliferative effects of cyclosporin in vitro. This is in line with our previous studies showing a range of sensitivity of T-lymphocytes to the antiproliferative effects of cyclosporin in other groups of patients with chronic severe asthma [4, 8]. Although these patients also showed a range of improvement in lung function when receiving cyclosporin therapy as compared with placebo, no relationship was observed between this clinical response and the sensitivity of the patients' T-lymphocytes to inhibition by cyclosporin in vitro. This proliferation assay is, therefore, not predictive of the degree of clinical response to oral cyclosporin therapy in vivo.

T-lymphocyte sensitivity both to glucocorticoids and cyclosporin in a similar assay was shown to correlate with outcome of renal allograft rejection and survival in patients with chronic renal failure [9]. In our clinical trial of cyclosporin therapy in asthma [5], no associations were observed between clinical response and variables such as age, atopic status, baseline lung function, or duration of disease. An in vitro assay to identify, prospectively, patients more (or less) likely to respond clinically to cyclosporin therapy might, therefore, have been a useful screening test and assessment of its possible value was the main objective of this study. These findings are in contrast to our previous study [3] of glucocorticoids, which suggested that asthmatic patients clinically refractory to oral prednisolone therapy in vivo have peripheral blood T-lymphocytes which are relatively resistant to the antiproliferative effects of dexamethasone in vitro. As the patients in the present study were receiving long-term oral prednisolone, it was not possible to measure their changes in lung function following a 2 week course of prednisolone, a test which we previously used to define clinical glucocorticoid sensitivity or resistance [3]. Although some individuals who showed clinical

b)

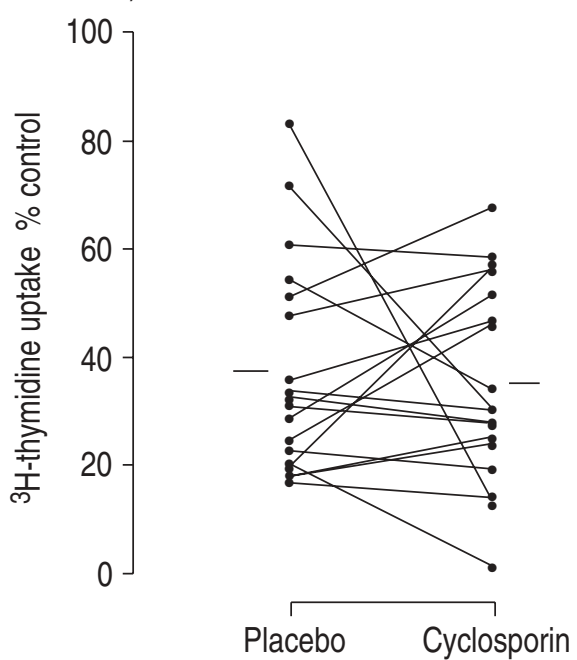

Fig. 2. - Comparison of the inhibition of PHA-induced proliferation of peripheral blood T-lymphocytes from patients with chronic severe asthma by: a) cyclosporin $\left(250 \mathrm{ng} \cdot \mathrm{mL}^{-1} ; \mathrm{n}=19\right)$; and b) dexamethasone $\left(10^{-7} \mathrm{~mol} \cdot \mathrm{L}^{-1} ; \mathrm{n}=18\right)$ while receiving placebo and cyclosporin therapy (see text). ${ }^{3} \mathrm{H}$-thymidine uptake (counts per minute) is expressed as a percentage of that measured in the presence of medium (no drug) control. Horizontal bars represent means. PHA: phytohaemagglutinin. 
improvement during cyclosporin therapy had peripheral blood T-lymphocytes which were relatively resistant to the antiproliferative effects of dexamethasone in vitro, suggesting that they might have been clinically resistant to glucocorticoid therapy, we were unable to confirm this and, thus determine whether asthmatic patients clinically resistant to glucocorticoids might be clinically sensitive to cyclosporin therapy.

A number of points may be relevant to the observed absence of an association between the in vivo effects of cyclosporin in ameliorating asthma and its in vitro effects on T-lymphocyte function in this assay.

Firstly, although two subsequent controlled clinical trials have shown cyclosporin to be efficacious in similar groups of patients $[10,11]$, the mechanism by which cyclosporin exerts its antiasthma effect is unknown. It is possible that inhibitory actions on other inflammatory cells, such as mast cells [12] and eosinophils [13], are involved. However, cyclosporin is believed to exert its immunosuppressive action principally by inhibiting $\mathrm{T}$ lymphocyte activation [14, 15], and we have recently shown that the clinical response of the same group of patients to cyclosporin therapy was associated with a reduction in their serum concentrations of soluble interleukin-2 receptor [16], a marker of T-lymphocyte activation.

Secondly, since the mechanism of any action of cyclosporin on T-lymphocytes which results in an antiasthma effect is unknown, it is possible to hypothesize that modulation of cytokine elaboration or function by cyclosporin may be more relevant than inhibition of $\mathrm{T}$ lymphocyte proliferation in this regard, and that cytokine production is not necessarily related to cell proliferation. Inhibition by cyclosporin of production by T-lymphocytes of cytokines relevant to asthma pathogenesis may, therefore, be a more appropriate predictor of its clinical efficacy than a proliferation assay. However, we had previously shown, using the same proliferation assay, that in vitro sensitivity to glucocorticoids was related to clinical response to prednisolone [3], and as the aim of the present study was to investigate whether the same relationship existed with cyclosporin, we used the same assay protocol.

Thirdly, a possible relationship may have been obscured by the following confounding factors. The patients were receiving variable dosages of oral prednisolone and their T-lymphocytes showed a wide range of sensitivity to the antiproliferative effects of glucocorticoids in vitro (fig. 2b). Although, as in a previous study [4], we failed to demonstrate a direct relationship between the antiproliferative effects of glucocorticoids and cyclosporin on peripheral blood T-lymphocytes from these asthmatic patients, this does not exclude the possibility of some degree of interaction of their antiasthma effects in vivo. This factor may be relevant, despite the fact that we excluded from the study those patients receiving dosages of prednisolone higher than their maintenance dosage at the time of blood sampling during disease exacerbations.

In addition, in vitro T-lymphocyte sensitivity to antiproliferative drugs may be subject to short-term variability. In the case of glucocorticoid sensitivity, we previously showed that our in vitro assay is highly reproducible [3, $8]$, both in terms of the coefficient of variability of repeated assays on the same subjects and also in the longer term, if the patients are retested after 4-6 months. Furthermore, T-lymphocytes from asthmatic patients were shown to be consistently sensitive or resistant to dexamethasone in vitro. We have similarly analysed the variability of repeated measurements of the inhibition of PHA-induced T-lymphocyte proliferation by cyclosporin in vitro in a group of seven normal subjects (data not show). Paired measurements of the cyclosporin concentration inhibiting $50 \%$ of the proliferation observed in the absence of drug (IC50) were made at intervals of $7-14$ days and the cyclosporin $\log _{10}$ IC50 compared, as described for dexamethasone [8]. The coefficient of variability of the logarithms of the differences in IC50 values measured on the two occasions was $10.5 \%$. Thus, the repeatability of the proliferative assay itself, when not potentially confounded by the effects of superadded therapy, is high. In this regard, it should be noted that the paired measurements shown in figure $2 \mathrm{a}$ do not necessarily reflect the inherent repeatability of the assay, since they were made in the presence and absence of cyclosporin therapy.

Although concomitant cyclosporin therapy of the asthmatic patients as a group did not result in any statistically significant change in the susceptibility of their peripheral blood T-lymphocytes both to cyclosporin and dexamethasone inhibition in vitro (in contrast to observations [17] on the inhibition by cyclosporin of mediator release from basophils ex-vivo), it may still have influenced these paired measurements within individuals. Similarly, it is possible that concomitant, variable oral prednisolone therapy may have influenced T-lymphocyte sensitivity to cyclosporin inhibition in vitro in certain individuals. Nevertheless, although there may be some interaction of the activities of cyclosporin and glucocorticoids in ameliorating asthma, our data suggest that this is apparently not evident in terms of any consistent antiproliferative effect in vitro on peripheral blood $\mathrm{T}$ lymphocytes.

Finally, the lack of predictive value of the in vitro assay may reflect the fact that inhibitory effects of cyclosporin on T-lymphocytes within the bronchial mucosa, which might be more relevant to the improvement of clinical asthma, differ from those on peripheral blood $\mathrm{T}$ lymphocytes. This possibility may be clarified further in future, if preparations of cyclosporin suitable for topical application to the bronchial mucosa become available. Indeed, it may then be possible to develop a short-term clinical predictive test for the antiasthma efficacy of cyclosporin, similar to that which we developed for glucocorticoids [3]. At present, in the case of orally administered cyclosporin, data from our existing trial [5] showed that the clinical response to cyclosporin therapy was not rapid, but progressive over the 12 week duration of the trial. Thus, any clinical trial of oral cyclosporin therapy might have to be conducted over a period of at least 12 weeks, limiting its usefulness as a "screening test". Furthermore, it was shown that clinical responsiveness to cyclosporin therapy could not be predicted by an exhaustive range of clinical variables, including age, gender, atopic status, baseline lung function and disease duration. It seems likely, therefore, that reliable tests for the antiasthma efficacy of cyclosporin must await more relevant in vitro tests and/or the possibility of assessment of topical therapy, which might produce more clear-cut 
and rapid clinical responses, with the additional advantage of a higher benefit/risk ratio.

Acknowledgements: The authors thank the patients, A Mandviwala, J. Flowers, A.J.M. Ward and A.F. Haczku for technical assistance, and Sandoz Pharmaceuticals for gifts of cyclosporin oily suspension and vehicle. A.G.A. was the 1989/1990 British Medical Association TV James Fellow for research into bronchial asthma.

\section{References}

1. Barnes PJ. A new approach to the treatment of asthma. N Engl J Med 1989; 321: 1517-1527.

2. Carmichael J, Patterson IC, Diaz P, Crompton GK, Kay $\mathrm{AB}$, Grant IWB. Corticosteroid resistance in chronic asthma. BMJ 1981; 282: 1419-1422.

3. Corrigan CJ, Brown PH, Barnes NC, et al. Glucocorticoid resistance in chronic asthma: glucocorticoid pharmacokinetics, glucocorticoid receptor characteristics, and inhibition of peripheral blood T-cell proliferation by glucocorticoids in vitro. Am Rev Respir Dis 1991; 144: 1016-1025.

4. Corrigan CJ, Brown PH, Barnes NC, Tsai JJ, Frew AJ, Kay AB. Glucocorticoid resistance in chronic asthma: peripheral blood T-lymphocyte activation and a comparison of the T-lymphocyte inhibitory effects of glucocorticoids and cyclosporin A. Am Rev Respir Dis 1991; 144: 1026-1032.

5. Alexander AG, Barnes NC, Kay AB. Trial of cyclosporin in corticosteroid-dependent chronic severe asthma. Lancet 1992; 339: 324-328.

6. Corrigan CJ, Kay AB. CD4 T-lymphocyte activation in acute severe asthma: relationship to disease severity and atopic status. Am Rev Respir Dis 1990; 141: 970-977.

7. Corrigan CJ, Haczku A, Gemou-Engesaeth V, et al. CD4 T-lymphocyte activation in asthma is accompanied by increased serum concentrations of interleukin-5: effect of glucocorticoid therapy. Am Rev Respir Dis 1993; 147: $540-547$.
8. Haczku A, Alexander AG, Brown P, et al. The effect of dexamethasone, cyclosporin and rapamycin on T-lymphocyte proliferation in vitro: comparison of cells from patients with glucocorticoid-sensitive and glucocorticoidresistant chronic asthma. J Allergy Clin Immunol 1994; 93: 510-519.

9. Pollak R, Dumble LJ, Lazda VA, Maddux MS, Stormoen $\mathrm{B}$, Ward M. Utility of an in vitro immunoassay to guide immunosuppressive therapy. Transplant Proc 1991; 23(1): 1113-1114.

10. Nizankowska E, Soja J, Pinis G, et al. Treatment of steroid-dependent bronchial asthma with cyclosporin. Eur Respir J 1995; 8: 1091-1099.

11. Lock SH, Kay AB, Barnes NC. Double-blind, placebocontrolled study of cyclosporin A as a corticosteroid sparing agent in corticosteroid-dependent asthma. Am J Respir Crit Care Med 1996; 153: 509-514.

12. Stellato C, de Paulis A, Ciccarelli A, et al. Anti-inflammatory effect of cyclosporin A on human skin mast cells. J Invest Dermatol 1992; 98: 800-804.

13. Kita H, Ohnishi T, Okubo Y, Weiler D, Abrams JS, Gleich GJ. Granulocyte/macrophage colony-stimulating factor and interleukin-3 release from human peripheral blood eosinophils and neutrophils. J Exp Med 1991; 174: 745-748.

14. Sigal NH, Dumont FJ. Cyclosporin A, FK-506, and rapamycin: pharmacological probes of lymphocyte signal transduction. Ann Rev Immunol 1992; 10: 519-560.

15. Granelli-Piperno A, Keane M, Steinman RM. Evidence that cyclosporin inhibits cell-mediated immunity primarily at the level of the T-lymphocyte rather than the accessory cell. Transplantation 1988; 46 (Suppl.) 53s-60s.

16. Alexander AG, Barnes NC, Kay AB, Corrigan CJ. Clinical response to cyclosporin in chronic severe asthma is associated with reduction in serum soluble interleukin-2 receptor concentrations. Eur Respir J 1995; 8: 574-578.

17. Casolaro V, Spadaro G, Patella V, Marone G. In vivo characterization of the anti-inflammatory effect of cyclosporin A on human basophils. J Immunol 1993; 151: 5563-5573. 